\title{
Fresnel-Kirchhoff integral for 2-D and 3-D path loss in outdoor urban environments
}

\author{
Ying Xu, Qiwu Tan, Member, IEEE, Danilo Erricolo, Senior Member, IEEE, \\ and Piergiorgio L. E. Uslenghi, Fellow, IEEE
}

\begin{abstract}
A deterministic model for propagation in outdoor urban environments based on the Fresnel-Kirchhoff integral is presented. Both two-dimensional (2-D) and three-dimensional (3-D) formulations considering reflection and diffraction are given. The theoretical predictions are compared with $2-\mathrm{D}$ and 3-D measurements and good agreement is achieved. The 3-D experiments, conducted in an anechoic chamber with scaled models representing buildings, are described in detail and provide a benchmark for comparisons with other methods.
\end{abstract}

Index Terms-Fresnel-Kirchhoff integral, measurements, urban environment, radio propagation, wireless communications.

\section{INTRODUCTION}

$\mathbf{T}$ HERE are three broad classes of methods to predict path loss in wireless communications: empirical [1], [2], statistical [3], [4], and deterministic models [5], [6], [7]. Deterministic models make use of detailed information about topography, buildings and, therefore, achieve more accurate results compared to empirical and statistical models. Deterministic models also provide clear physical insights into various effects such as reflection, diffraction and scattering from buildings or terrain. However, one drawback of deterministic models is that they tend to be more complicated and time-consuming than empirical and statistical models.

Most deterministic models for outdoor environments are either based on ray-tracing algorithms [5], [6], [7], [8] or field integral methods [9], [10], [11]. On one hand, raytracing algorithms are more popular because they are easy to implement, and relatively fast since they are based on high-frequency asymptotic solutions. On the other hand, field integral methods are potentially more accurate.

This paper is about the application of the Fresnel-Kirchhoff integral that belongs to the class of deterministic, field integral based methods.

The Fresnel-Kirchhoff integral was introduced in optics to mathematically formulate diffraction based upon Huygens' principle. More recently, it was applied to obtain accurate evaluations of radio communication links to estimate diffraction past obstacles. For this purpose, the simplest way to apply the Fresnel-Kirchhoff integral is to refer to a geometry, such as an irregular terrain or a hill, where the obstacle may

Ying $\mathrm{Xu}$, Danilo Erricolo, and Piergiorgio L. E. Uslenghi are with the Department of Electrical and Computer Engineering, University of Illinois at Chicago, Chicago, IL 60607-7053 USA (email: erricolo@ece.uic.edu, uslenghi@uic.edu). Qiwu Tan is with Amphenol T\&M Antennas (qtan@tmantennas.com). This research was supported in part by a grant from the Andrew Foundation. be approximated as an infinitely wide two-dimensional (2-D) single knife edge [12].

Let us review some applications of the Fresnel-Kirchhoff integral to investigate 2-D geometries. Millington et al. [13] extended the use of Fresnel-Kirchhoff integrals to double knife edges. Based on the results of Furutsu [14], Vogler [15] developed a numerical method to compute diffraction from multiple knife edges. He evaluated the multidimensional integral using a repeated integral of the error function; unfortunately, the time required for the evaluation of the integral limited the number of knife edges that could be treated with this method. Whitteker [10] improved the results of Vogler by connecting adjacent knife edges with reflecting surfaces and included the effect of reflection in the formulation of the Fresnel-Kirchhoff integral. In particular, Whitteker adopted the numerical integration technique of Stamnes [16] and computed the multiple integral in steps proceeding from one knife edge to the successive one. With the fast development of modern wireless communications, the Fresnel-Kirchhoff integral became a useful tool to predict propagation path loss caused by buildings in urban and suburban areas. As an example, Walfisch and Bertoni [17] approximated buildings as infinitely-wide absorbing half screens of uniform height and spacing, and used the Fresnel-Kirchhoff integrals to compute the multiple forward diffraction.

Three-dimensional (3-D) geometries have also been considered. Assis [18] examined an obstacle that was approximated as a single knife edge of finite size. Savov et al. [19] and Haslett [20] applied the Fresnel-Kirchhoff integral to determine the field strength behind a 3-D building. In the model of Russell et al. [21], the Fresnel-Kirchhoff theory was extended to a general case where buildings could be arbitrarily located and oriented in any direction; however, reflection was not included.

In addition to the Fresnel-Kirchhoff integral, there are other field integral based methods such as the parabolic equation (PE) in integral form solved by split-step Fourier transform [9], [22], [23], the path integral technique [11], [24], the solution of electric field integral equation (EFIE) [25] and magnetic field integral equation (MFIE) [26], [27]. The PE method also obtains the field strength by applying a marching algorithm. The path integral was originally used by Feynman $e t$ al. [28] in quantum mechanics. Lee [29] employed it to solve the problem of multiple knife edge diffraction. In [30], it was applied to 2-D and 3-D urban environments, and the multi-dimensional integral was computed by repeated integral of error functions. Although the Fresnel-Kirchhoff integral and the path integral 


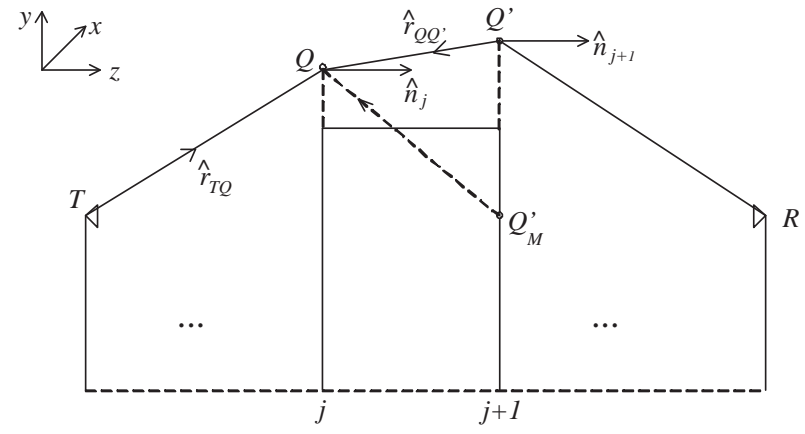

Fig. 1. Geometry for two obstacles approximated with two infinitely-wide knife edges

have different origins, their final expressions are equivalent.

In this paper, the Fresnel-Kirchhoff integral is used to predict field strength in outdoor urban environments. Diffraction at edges and reflections from roofs and walls are taken into account. The formulations of both 2-D and 3$\mathrm{D}$ cases are given and comparisons with measurements are provided. The 3-D formulations developed in this paper not only account for reflection and diffraction, but also apply to general environments where buildings have arbitrary locations and orientations. In the integral, an angle factor neglected by other models is kept and therefore more accurate results can be achieved.

For the 2-D case, the measurement results are taken from D'Elia et al. [31] and Crovella et al. [32], who assessed the accuracy of the 2-D polygonal line ray-tracing simulator of Erricolo and Uslenghi [8]. For the 3-D case, part of the measurements was reported by $\mathrm{Xu}$ et al. in [33] while the rest is presented in this work. Both 2-D and 3-D experiments were carried out in the anechoic chamber at the University of Illinois at Chicago on scaled models of buildings coated with copper sheets. The advantage of using an anechoic chamber is that most unwanted reflections and noise sources are removed and the parameters are all known [34].

This paper provides detailed documentation of the 3-D measurements, which is important because it provides a benchmark for other researchers who want to compare their methods with experimental results. In fact, to the best of our knowledge, only a limited number of other works [11], [30], [35] provide sufficient details of 3-D measurements to allow for a comparison with theoretical methods.

\section{TWO-DIMENSIONAL SCENARIO}

\section{A. Formulation}

The 2-D scenario is discussed first to make the 3-D case easier to understand. Since analytical solutions are only available for one or two knife edges [13], numerical methods have to be used for three or more obstacles.

Let us consider the geometry shown in Fig. 1 where multiple infinitely wide knife edges are present between the transmitter and receiver. Similar to Whitteker's derivation[10], the $y$ dependence $E_{y}\left(Q^{\prime}\right)$ of the scalar field at point $Q^{\prime}\left(x_{Q^{\prime}}, y_{Q^{\prime}}, z_{Q^{\prime}}\right)$ in the aperture of knife edge $j+1$ can be obtained from the fields of all points $Q\left(x_{Q}, y_{Q}, z_{Q}\right)$ above knife edge $j$ (for more detailed derivation, see [36], [37]):

$$
\begin{aligned}
& E_{y}\left(Q^{\prime}\right)=\frac{-i k}{2 \pi} \int_{-\infty}^{+\infty} E_{x}(Q) e^{i k \frac{\left(x_{Q^{\prime}}-x_{Q}\right)^{2}}{2\left(z_{Q^{\prime}}-z_{Q}\right)}} d x_{Q} \\
& \cdot \int_{h_{j}}^{\infty}\left[E_{y}(Q) \frac{e^{i k \sqrt{\left(y_{Q}-y_{Q^{\prime}}\right)^{2}+\left(z_{Q}-z_{Q^{\prime}}\right)^{2}}}}{\sqrt{\left(y_{Q}-y_{Q^{\prime}}\right)^{2}+\left(z_{Q}-z_{Q^{\prime}}\right)^{2}}} \Psi_{j}\right. \\
& \left.+R_{F} E_{y}(Q) \frac{e^{i k \sqrt{\left(y_{Q}-y_{Q_{M}^{\prime}}\right)^{2}+\left(z_{Q}-z_{Q_{M}^{\prime}}\right)^{2}}}}{\sqrt{\left(y_{Q}-y_{Q_{M}^{\prime}}\right)^{2}+\left(z_{Q}-z_{Q_{M}^{\prime}}\right)^{2}}} \Psi_{j}^{\prime}\right] d y_{Q},
\end{aligned}
$$

where $x_{Q^{\prime}}=x_{T} ; k$ is the wave number; $h_{j}$ is the height of knife edge $j$; and $R_{F}$ is the reflection coefficient if knife edge $j$ and $j+1$ are connected by a reflecting surface. $Q_{M}^{\prime}\left(x_{Q_{M}^{\prime}}, y_{Q_{M}^{\prime}}, z_{Q_{M}^{\prime}}\right)$ is the image of $Q^{\prime}$ with respect to the reflecting surface;

$$
\begin{aligned}
& \Psi_{j}=\left[\cos \left(\hat{n}_{j}, \hat{r}_{T Q}\right)-\cos \left(\hat{n}_{j}, \hat{r}_{Q Q^{\prime}}\right)\right] / 2, \\
& \Psi_{j}^{\prime}=\left[\cos \left(\hat{n}_{j}, \hat{r}_{T Q}\right)-\cos \left(\hat{n}_{j}, \hat{r}_{Q Q_{M}^{\prime}}\right)\right] / 2,
\end{aligned}
$$

where $\hat{n}_{j}$ is the unit normal vector of the aperture above knife edge $j ; \hat{r}_{T Q}, \hat{r}_{Q Q^{\prime}}$ and $\hat{r}_{Q Q_{M}^{\prime}}$ are the unit vectors from $T$ to $Q, Q^{\prime}$ to $Q$ and $Q_{M}^{\prime}$ to $Q$, respectively; $E_{x}(Q)$ and $E_{y}(Q)$ are the $x$ and $y$ dependences of the field $E(Q)$,

$$
E_{x}(Q)=e^{i k \frac{\left(x_{Q}-x_{T}\right)^{2}}{2\left(z_{Q}-z_{T}\right)}}
$$

$E_{y}(Q)$ has to be computed step by step, but for the point $Q_{1}$ above the first knife edge

$$
E_{y}\left(Q_{1}\right)=\frac{e^{i k \sqrt{\left(y_{Q_{1}}-y_{T}\right)^{2}+\left(z_{Q_{1}}-z_{T}\right)^{2}}}}{\sqrt{\left(y_{Q_{1}}-y_{T}\right)^{2}+\left(z_{Q_{1}}-z_{T}\right)^{2}}} .
$$

Applying (1) repeatedly, the field at the receiver can be computed. The normalized field is defined as the magnitude of the received field $E(R)$ divided by the free space (or incident) field $E_{\text {free }}$ at the receiver and expressed in $\mathrm{dB}$ :

$$
E_{0}(R)=20 \log \left|\frac{E(R)}{E_{\text {free }}(R)}\right|(\mathrm{dB}) .
$$

Other models using Fresnel-Kirchhoff integral [10], [19], [21] neglect the angle factors $\Psi_{j}, \Psi_{j}^{\prime}$ and approximate them with unity. This can lead to errors when the angles are not small. To reduce the error, Savov et al. [19] introduced an extra wide-angle correction factor at the receiver. In this paper, the angle factors are kept in the integrals, so no correction factor is needed and the results should be more accurate.

In (1), the integral in the first factor can be transformed to the well-known Fresnel integral. However, the integral in the second factor is difficult to evaluate. So, after Whitteker [10], the numerical technique of Stamnes et al. [16] is applied for the computation of this integral.

\section{B. Experimental validation}

The experimental validation for the 2-D scenario is carried out using the data obtained from the experiments described in [31]. These experiments are carefully designed to satisfy the 2-D approximation by using special high gain sector antennas, sufficiently wide scaled models of buildings made of 
copper sheets and by taking measurements inside an anechoic chamber. The operating frequency is $25 \mathrm{GHz}$.

Referring to Fig. 2(a), two buildings are placed between the transmitter and the receiver. The receiver is moved vertically from shadow to line-of-sight (LOS) region. Since the buildings and the ground are made of copper sheets, both reflection and diffraction have to be considered. The reflection coefficient from the ground and buildings is +1 for vertical polarization and -1 for horizontal polarization. In Fig. 2(b), the normalized field computed using the Fresnel-Kirchhoff integral is compared with the measurements for vertical polarization. In the shadow region, the field suffers strong attenuation and fluctuates rapidly, while in the LOS region the field varies much more smoothly. The mean error between the measurements and the theoretical prediction is $-1.15 \mathrm{~dB}$ and the standard deviation is $1.38 \mathrm{~dB}$. Results for horizontal polarization are illustrated in Fig. 2(c). The mean error is $0.26 \mathrm{~dB}$ and the standard deviation is $1.28 \mathrm{~dB}$. Therefore, the good agreement with experiments for both polarizations validates the Fresnel-Kirchhoff integral formulation in two dimensions.

\section{THREE-DIMENSIONAL SCENARIO}

\section{A. Formulation}

As illustrated in Fig. 3, two finite-width knife edges are located between the transmitter and receiver. The field at the receiver is composed of three parts: the contributions from the top, left and right sides of both knife edges.

First consider the diffraction from the top of the two knife edges. The $y$ dependence of the field at point $Q^{\prime}$ in the aperture above knife edge $j+1$ is the same as in (1) except that $x_{Q}$ is integrated from $x_{j-}$ to $x_{j+}$, which are the $x$ coordinates of the right and left sides of knife edge $j$, respectively. From $E_{y}\left(Q^{\prime}\right), E_{\text {top }}(R)$ can be computed accordingly.

The diffraction from the sides of the two knife edges can be obtained similarly. For the vertical edges, the roles of $x$ and $y$ should be interchanged in the formulations. On the right side of knife edge $j+1$, the $x$ dependence of $E\left(P^{\prime}\right)$ can be written as

$$
\begin{aligned}
& E_{x}\left(P^{\prime}\right)=\frac{-i k}{2 \pi} \int_{-\infty}^{+\infty} E_{y}(P) e^{i k \frac{\left(y_{P^{\prime}}-y_{P}\right)^{2}}{2\left(z_{P^{\prime}}-z_{P}\right)}} d y_{P} \\
& \cdot \int_{-\infty}^{x_{j}-}\left[E_{x}(P) \frac{e^{i k \sqrt{\left(x_{P}-x_{P^{\prime}}\right)^{2}+\left(z_{P}-z_{P^{\prime}}\right)^{2}}}}{\sqrt{\left(x_{P}-x_{P^{\prime}}\right)^{2}+\left(z_{P}-z_{P^{\prime}}\right)^{2}}} \Psi_{r j}\right. \\
& \left.+R_{r F} E_{x}(P) \frac{e^{i k \sqrt{\left(x_{P}-x_{P_{M}^{\prime}}\right)^{2}+\left(z_{P}-z_{P_{M}^{\prime}}\right)^{2}}}}{\sqrt{\left(x_{P}-x_{P_{M}^{\prime}}\right)^{2}+\left(z_{P}-z_{P_{M}^{\prime}}\right)^{2}}} \Psi_{r j}^{\prime}\right] d x_{P},
\end{aligned}
$$

where $y_{P^{\prime}}=y_{T} ; P_{M}^{\prime}$ is the image of $P^{\prime}$ if there is a vertical reflecting surface connecting the two edges with reflection coefficient $R_{r F} ; \Psi_{r j}$ and $\Psi_{r j}^{\prime}$ are the same as $\Psi_{j}$ and $\Psi_{j}^{\prime}$ in (2) and (3) with $Q, Q^{\prime}$ and $Q_{M}^{\prime}$ replaced by $P, P^{\prime}$ and $P_{M}^{\prime}$;

$$
\begin{aligned}
& E_{x}(P)=\frac{e^{i k \sqrt{\left(x_{P}-x_{T}\right)^{2}+\left(z_{P}-z_{T}\right)^{2}}}}{\sqrt{\left(x_{P}-x_{T}\right)^{2}+\left(z_{P}-z_{T}\right)^{2}}} ; \\
& E_{y}(P)=e^{i k \frac{\left(y_{P}-y_{T}\right)^{2}}{2\left(z_{P}-z_{T}\right)}} .
\end{aligned}
$$

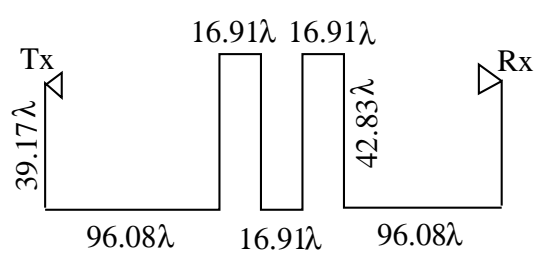

(a) Two-building profile

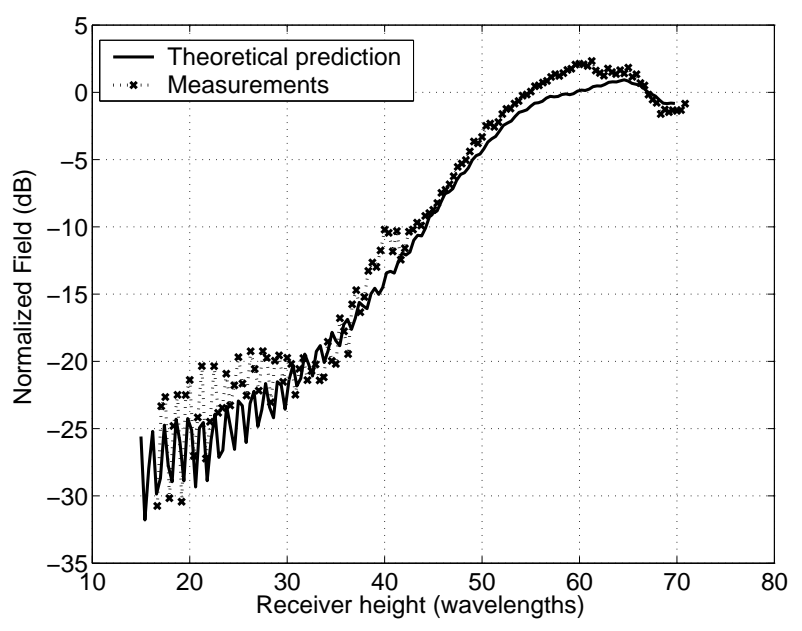

(b) Normalized field, vertical polarization

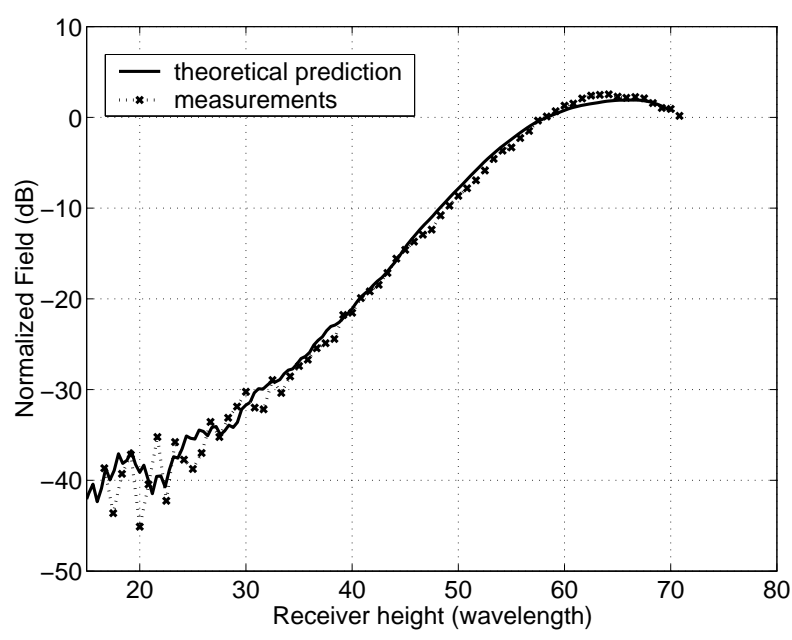

(c) Normalized field, horizontal polarization

Fig. 2. Geometry and normalized field for 2-D experimental validation

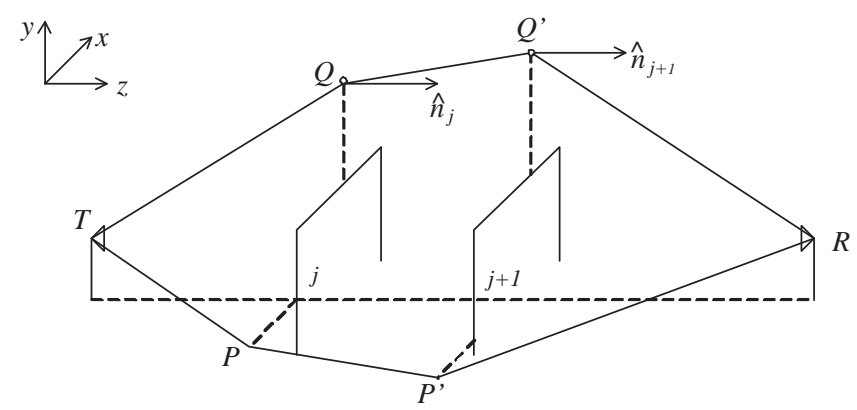

Fig. 3. Two finite size knife edges 


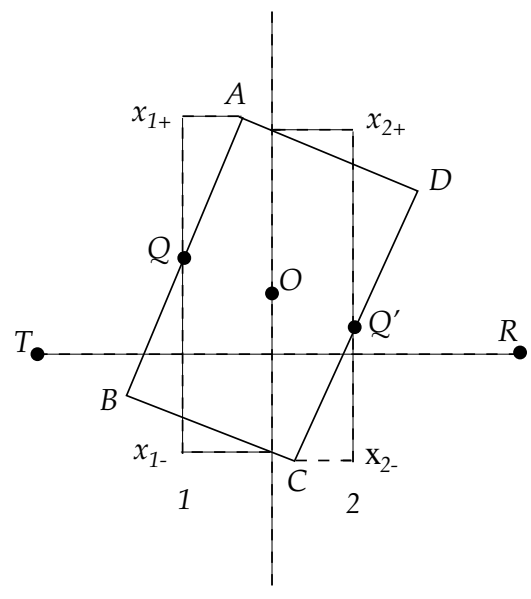

Fig. 4. Building not perpendicular to the line $T R$, top view

In (7) the ground is neglected, so $E_{y}(P)$ is integrated from $-\infty$ to $\infty$. Otherwise the lower limit should be the height of the ground.

Then $E_{\text {right }}(R)$ can be computed from $E_{x}\left(P^{\prime}\right)$. The contribution $E_{\text {left }}(R)$ from the left side of the knife edges can be obtained in the same way with appropriate change of the integration limits along the $x$ direction.

Finally the total field at the receiver is the sum of the above three parts,

$$
E(R)=E_{\text {top }}(R)+E_{\text {right }}(R)+E_{\text {left }}(R) .
$$

Cross terms such as contribution from the top of knife edge $j$ to the side of knife edge $j+1$, and vice versa, can also be computed. However, these terms are relatively small in magnitude and therefore neglected.

In real environments, it happens frequently that the faces of buildings are not perpendicular to the line connecting the transmitter and receiver, such as in the top view of Fig. 4. For this example, the contribution from the sides is computed as described earlier by evaluating diffraction and reflection at the corners $A, D$ on the left side and $B, C$ on the right side.

The contribution from the top needs a few more adjustments, because - in order to apply formulas such as (1) —one needs roof edges that are perpendicular to the direction $T R$. This is obtained by projecting the segments $A B$ and $C D$ onto the transversal planes passing through $Q$ and $Q^{\prime}$, which are the centers of $A B$ and $C D$, respectively. The projection of the segment $A B$ results into line 1, which is limited between $x_{1-}$ and $x_{1+}$. Similarly, the projection of the segment $C D$ produces line 2 , which is confined between $x_{2-}$ and $x_{2+}$. The lines 1 and 2 define two knife edges that are used to evaluate the contribution from the top.

For the general case of multiple buildings oriented randomly, one can get the overall field by applying the above algorithm repeatedly from the transmitter to the receiver.

\section{B. Experimental validation}

In order to discuss the experimental results, some clarifications must be made regarding the antennas used in the experiments and other details of the measurements. The transmitting and receiving antennas are assumed to be isotropic in the Fresnel-Kirchhoff formulation introduced so far. However, since no isotropic antennas actually exist, one has either to introduce the directivity in the theoretical formulation to characterize the antennas, or design experiments where the effect of the directivity is reduced as much as possible.

Accounting for the directivity in the theoretical formulation requires the introduction of an additional weighting factor in the integrals. However, one purpose of this work is to provide well documented 3-D measurement results so that they may be used by other researchers to make comparisons. Hence, instead of modifying the Fresnel-Kirchhoff formulation, the following measures are taken to reduce the directivity effect in the experiments.

1. The receiving antenna is an open-ended waveguide, which has a very weak directivity and thus a wide $3 \mathrm{~dB}$ beam-width in both $E\left(86^{0}\right)$ and $H\left(47.6^{0}\right)$ planes. The transmitter is a standard horn antenna. If the transmitter were also an openended waveguide, the received field would be very weak which could lead to larger error.

2. The receiver is located inside the $3 \mathrm{~dB}$ beam-width and pointed toward the direction of maximum radiation of the transmitter. The buildings are inside the $3 \mathrm{~dB}$ beam-width of the transmitter.

3. For the same purpose as above, the heights of the transmitter and receiver are almost the same.

4. In the experiments involving only one scaled building model of size $(35.55 \mathrm{~cm}, 35.55 \mathrm{~cm}, 28 \mathrm{~cm})$, the experimental and theoretical results are in good agreement. Unfortunately, due to the small size of the anechoic chamber used in the experiments, when two scaled models of this size are located one after the other between the two antennas, the effect of antenna directivity becomes more noticeable. Therefore, in order to reduce the angles involved when two buildings are present, one of them is made smaller than the other, and as a result, the agreement with the theory improves.

With the above special considerations, the antenna directivity no longer plays a major role in the measured results.

The measurements are taken on scaled models of buildings made with wood and covered with copper sheets, so reflection from roof and side walls must be accounted for. Reflection from the ground is neglected because the ground is covered with absorbing material. Transmitter and receiver initially face each other along a direction referred to as the longitudinal direction. At the beginning, the receiver is located in the shadow zone of the building. Then it is moved transversely with respect to the longitudinal direction and eventually reaches the LOS region of the transmitter. The operating frequency is $25 \mathrm{GHz}$. Since the wavelength is very short $(1.2 \mathrm{~cm})$, everything has to be laid out very accurately. A displacement of $1 \mathrm{~cm}$ (especially in transverse direction) can lead to considerable error. The maximum error in the experiments is $\pm 0.5 \mathrm{~dB}$.

The first set of measurements are about a single building located in different positions between the two antennas. Fig. 5(a) shows the geometry of the first measurements, with two faces of the building perpendicular to the longitudinal direction. The distance from the transmitter to the back face of the building 


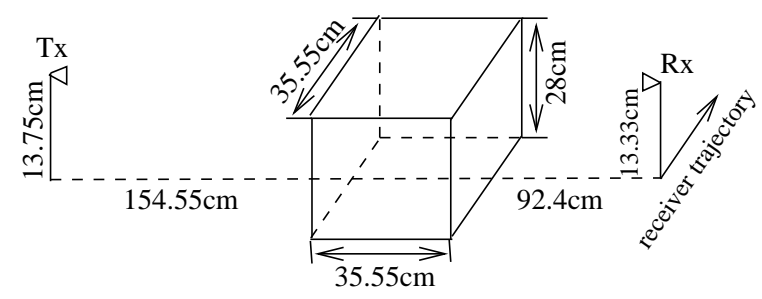

(a) One building block

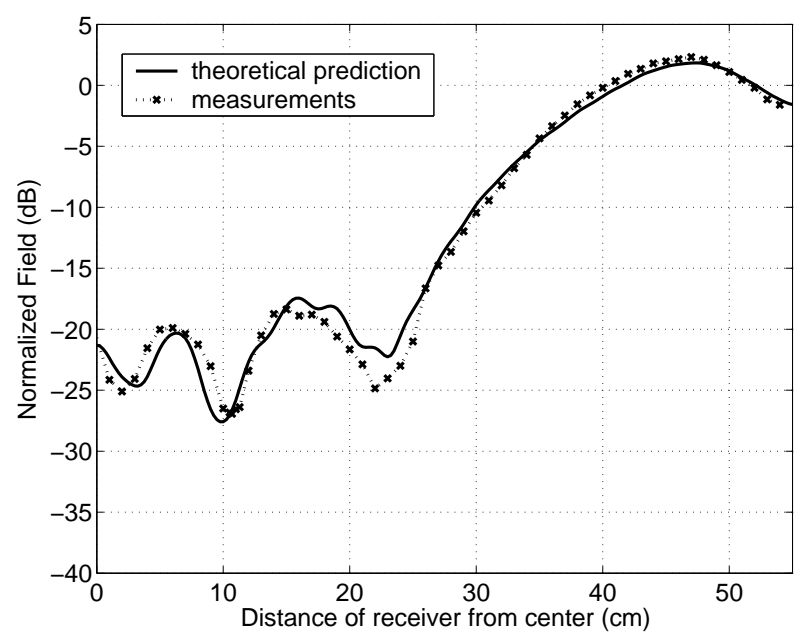

(b) Normalized field, vertical polarization

Fig. 5. Geometry and normalized field for a 3-D building

is $154.55 \mathrm{~cm}$; the distance from the receiver to the front face of the building is $92.4 \mathrm{~cm}$; the heights of the transmitter and receiver are $13.75 \mathrm{~cm}$ and $13.33 \mathrm{~cm}$, respectively. The measured results for vertical polarization are shown in Fig. 5(b) where they are compared with the theoretical prediction that is evaluated using reflection coefficients of +1 and -1 from the top and sides of the building, respectively. When the receiver is in the shadow region, the measured field is small in magnitude and shows two major oscillations due to the multipath effect. As the receiver moves towards the LOS region, the measured field increases smoothly and reaches a value around $0 \mathrm{~dB}$ as expected, due to the normalization. The mean error between the measurements and the theoretical prediction is $0.08 \mathrm{~dB}$ and the standard deviation is $1.23 \mathrm{~dB}$. The theoretical prediction is in good agreement with the measurements.

The geometry and field results for horizontal polarization are illustrated in Figs. 6(a) and 6(b). The reflection coefficients of the top and sides of the building are exchanged to -1 and +1 , respectively, in this case. Compared with Fig. 5(b), the field fluctuates more rapidly in the shadow region and the fluctuation amplitude is slightly stronger on average. The mean error between the measurements and the theoretical prediction is $-0.66 \mathrm{~dB}$ and the standard deviation is $2.36 \mathrm{~dB}$.

In Fig. 7 (a) the building is rotated $20^{\circ}$ clockwise horizontally around its center, so that its faces are no longer perpendicular to the longitudinal line from the transmitter to the receiver. The center of the building is $172.1 \mathrm{~cm}$ away from the transmitter and $110.4 \mathrm{~cm}$ from the receiver; the heights of the transmitter and receiver are $13.72 \mathrm{~cm}$ and $13.9 \mathrm{~cm}$. For this example, the algorithm illustrated in Fig. 4 is used.

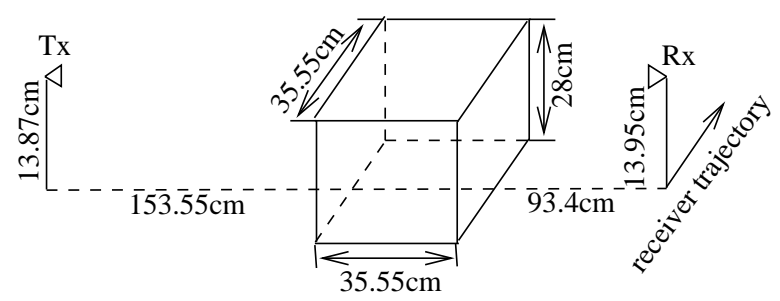

(a) One building block

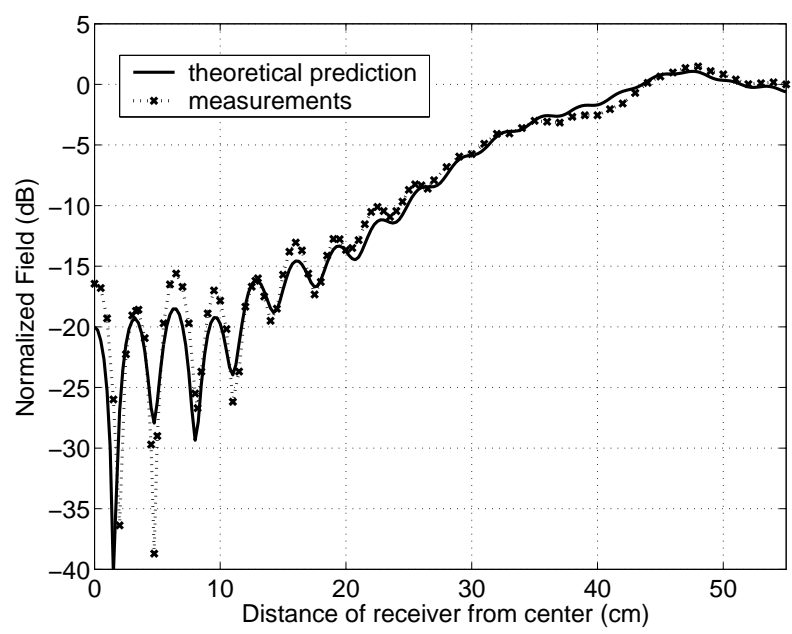

(b) Normalized field, horizontal polarization

Fig. 6. Geometry and normalized field for a 3-D building

The comparisons of theoretical results and measurements for vertical and horizontal polarizations are plotted in Figs. 7(b) and $7(\mathrm{c})$, respectively. For vertical polarization, the mean error is $-0.06 \mathrm{~dB}$ and the standard deviation is $2.05 \mathrm{~dB}$. For horizontal polarization, the mean error is $-0.1 \mathrm{~dB}$ and the standard deviation is $2.75 \mathrm{~dB}$. The good agreement in both cases validates the adjusted algorithm for this more general configuration.

Next, in Fig. 8(a), the building is rotated $45^{0}$ clockwise horizontally around its center. The distance from the transmitter to the back corner of the building is $140.02 \mathrm{~cm}$; and the receiver is $92.2 \mathrm{~cm}$ away from the front corner. Fig. 8(b) shows the top view of this setting. Similar to Fig. 4, the top contribution can be approximated as the diffraction and reflection from knife edges 1 and 2, and the left and right side fields come from the vertical edges $A, D, C$ and $A, B, C$, respectively.

The field for the vertical polarization is shown in Fig. 8(c). Notice that, since the building is rotated $45^{\circ}$, a longer portion of the receiver trajectory is in the shadow. So instead of the two ripples in Figs. 5(b) and 7(b) in the non-lit region, there are three ripples. The mean error is $-0.38 \mathrm{~dB}$ and the standard deviation is $2.71 \mathrm{~dB}$. The corresponding geometry and normalized field for horizontal polarization are plotted in Figs. 9 (a) and 9 (b). The mean error is $0.83 \mathrm{~dB}$ and the standard deviation is $2.68 \mathrm{~dB}$.

In Fig. 10(a), two buildings are present. For the reason explained earlier, one building is reduced in size to lessen the effect of antenna directivity. The transmitter is $100.15 \mathrm{~cm}$ from the back face of the small box; the distance between two 


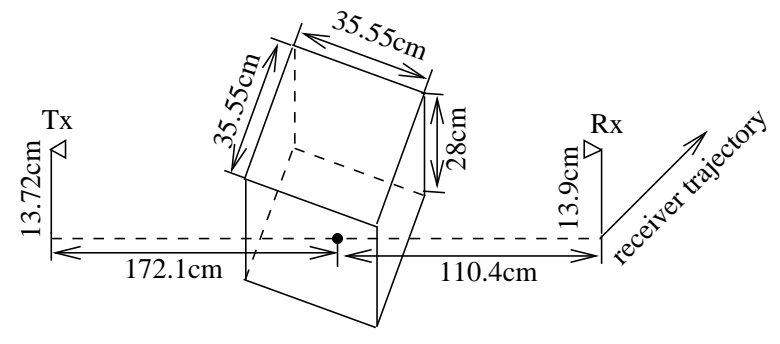

(a) One building block rotated $20^{0}$ clockwise

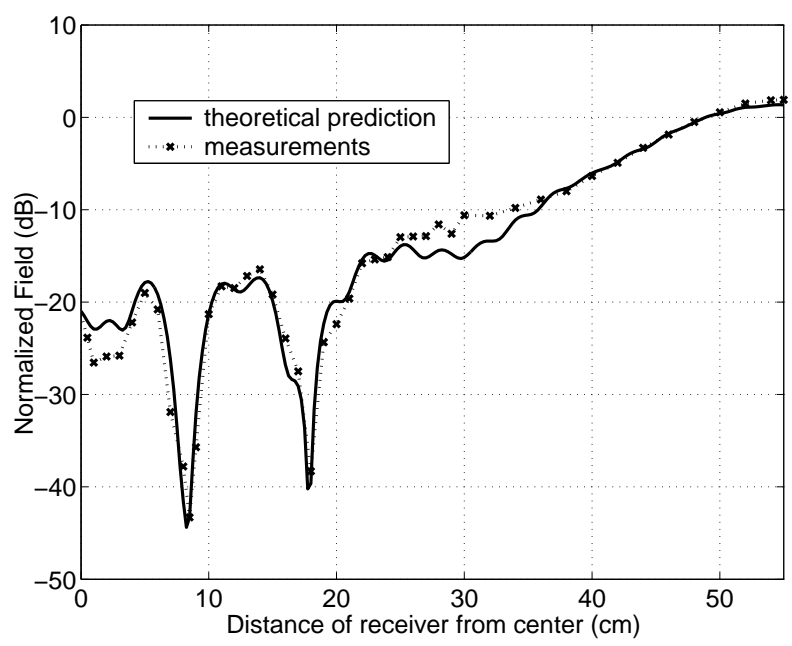

(b) Normalized field, vertical polarization

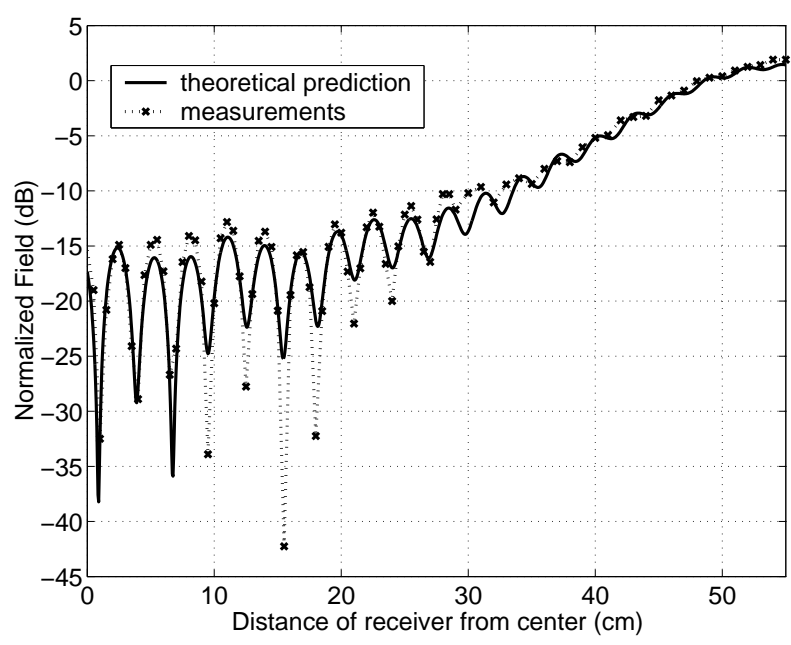

(c) Normalized field, horizontal polarization

Fig. 7. Geometry and normalized field for a building rotated $20^{0}$ clockwise

adjacent parallel faces of the two boxes is $43.2 \mathrm{~cm}$; the front face of the big box is $93.1 \mathrm{~cm}$ from the receiver. In the shadow region, the receiver now suffers double blockage from the two buildings, so the field is even smaller compared to Fig. 5(b). When the receiver moves away from the longitudinal axis, the measured field is affected more by the larger box than the smaller one because of their dimensions. Therefore the smaller box actually does not contribute much to the total field in the LOS region, as one can see by comparing Figs. 10(b) and 5(b). The mean error between theoretical prediction and measurements is $0.15 \mathrm{~dB}$ and the standard deviation is $2.01 \mathrm{~dB}$.

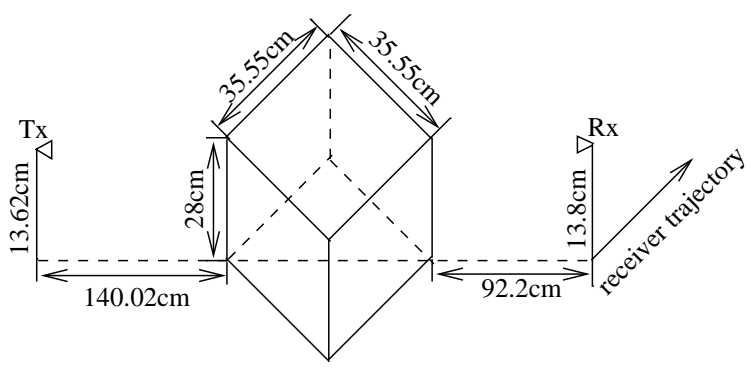

(a) One building block rotated $45^{0}$ clockwise

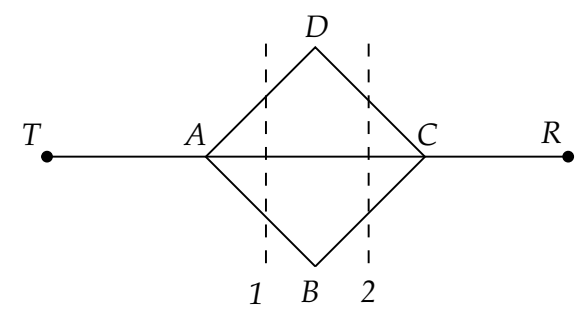

(b) top view of (a)

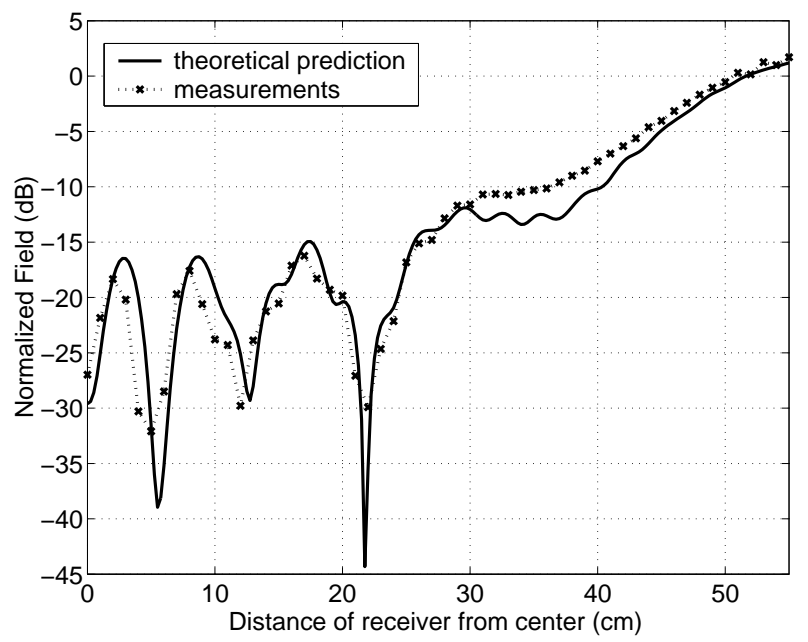

(c) Normalized field, vertical polarization

Fig. 8. Geometry and normalized field for a building rotated $45^{0}$ clockwise

The above analysis also applies to the horizontal polarization. The field in Fig. 10(c) is a few dBs lower than Fig. 6(b) in the shadow region. The mean error is $-1.95 \mathrm{~dB}$ and the standard deviation is $1.14 \mathrm{~dB}$. For the two-building setup, the theoretical results still agree well with the measurements.

Next only the small box is rotated $20^{0}$ clockwise horizontally, as shown in Fig. 11(a). The distances from the center of the small box to the transmitter and the back face of the big box are $101.95 \mathrm{~cm}$ and $51.5 \mathrm{~cm}$, respectively; the receiver is $93.5 \mathrm{~cm}$ from the front face of the big box. The theoretical predictions and measurements for vertical and horizontal polarizations are compared in Figs. 11(b) and 11(c). For vertical polarization, the mean error is $1.14 \mathrm{~dB}$ and the standard deviation is $2.48 \mathrm{~dB}$. For horizontal polarization, the mean error is $-0.88 \mathrm{~dB}$ and the standard deviation is $1.63 \mathrm{~dB}$.

Finally, both boxes are rotated $20^{\circ}$ clockwise, as shown in Fig. 12(a). The transmitter is $101.95 \mathrm{~cm}$ from the center of the small box; the distance between the centers of two boxes is 


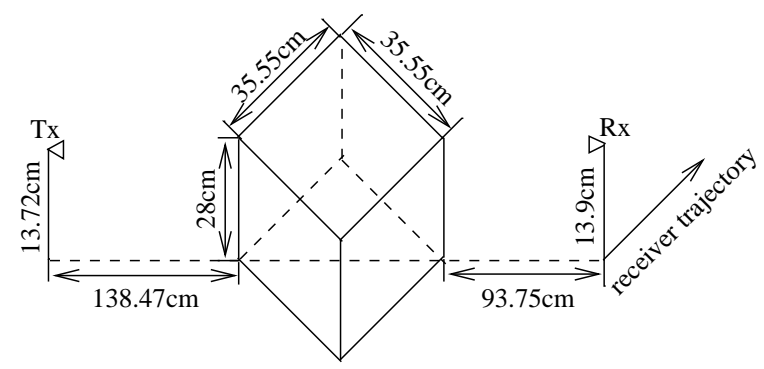

(a) One building block rotated $45^{0}$ clockwise

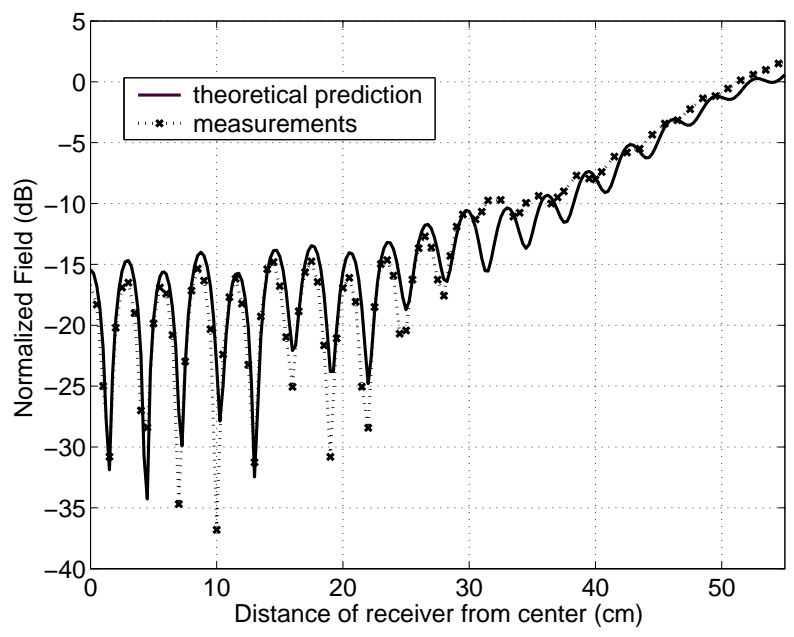

(b) Normalized field, horizontal polarization

Fig. 9. Geometry and normalized field for a building rotated $45^{0}$ clockwise

$68.37 \mathrm{~cm}$; the receiver is $112.18 \mathrm{~cm}$ from the center of the big box. For vertical polarization in Fig. 12(b), the mean error is $1.73 \mathrm{~dB}$ and the standard deviation is $2.08 \mathrm{~dB}$. For horizontal polarization in Fig. 12(c), the mean error is $0.2 \mathrm{~dB}$ and the standard deviation is $2.95 \mathrm{~dB}$.

\section{CONCLUSION}

The Fresnel-Kirchhoff integral is investigated to predict field strength in outdoor urban environments. The 2-D and 3-D formulations can account for both reflection and diffraction. In particular, the 3-D model can be applied to a general environment where buildings have arbitrary locations and orientations. In addition, an angle factor that is usually ignored by other models is kept inside the integral, which makes the result more accurate. Experiments are conducted inside an anechoic chamber to verify the theory. Buildings are represented by wooden scaled models coated with copper sheets. The biggest challenge of the 3-D experiments is to remove the directivity of antennas as much as possible, because the theory developed so far assumes that antennas are isotropic. Different configurations involving one and two buildings are considered, and the excellent agreement between the theoretical results and measurements for vertical and horizontal polarizations proves that the Fresnel-Kirchhoff integral is an accurate deterministic method for both 2-D and 3-D.

The experimental results presented in this paper will be useful for other researchers to compare their methods with our measurements.

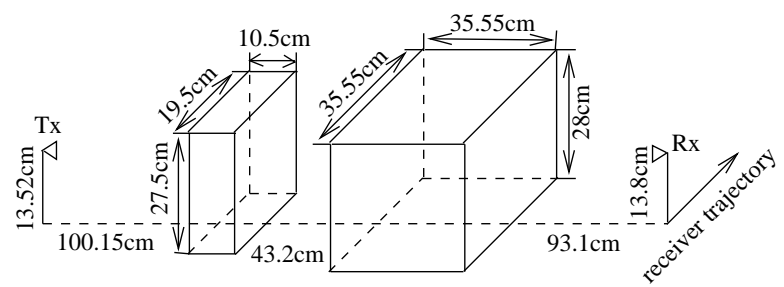

(a) Two-building block

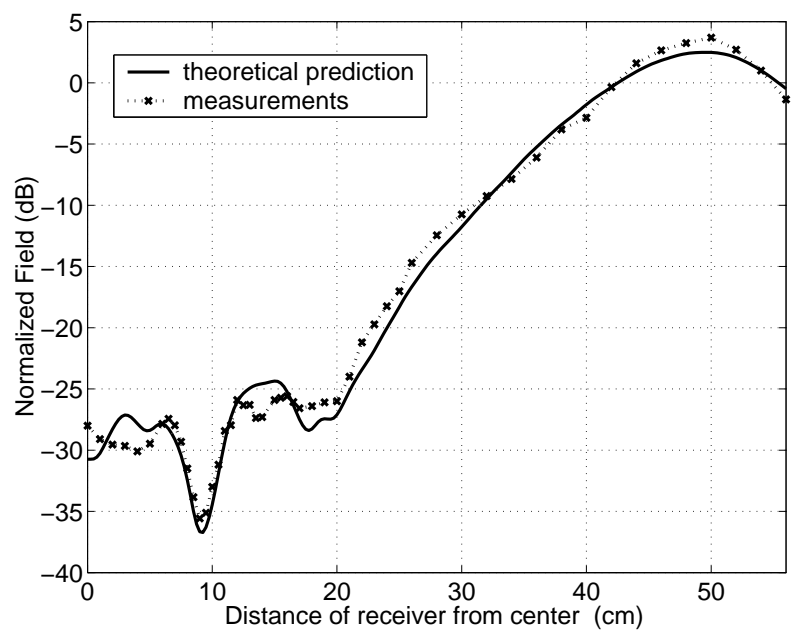

(b) Normalized field, vertical polarization

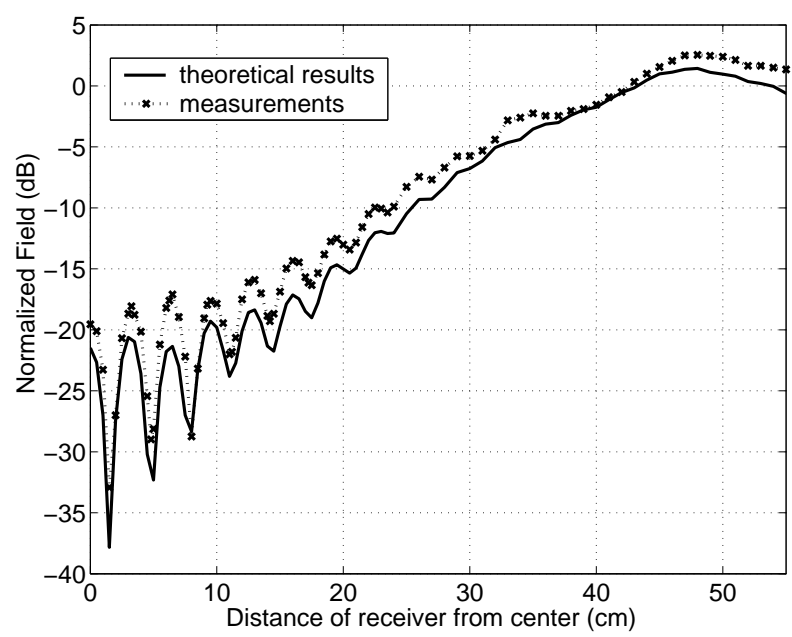

(c) Normalized field, horizontal polarization

Fig. 10. Geometry and normalized field for two buildings

In the experiments, the scaled models coated with copper sheets are an idealization of buildings of brick and concrete walls in real environments that have weaker reflection. Although the scaled models can not accurately represent the real buildings, they are useful to verify the theoretical method. Also for real antennas, a weighting factor of antenna directivity should be introduced in the Fresnel-Kirchhoff integral. In the future, more experiments should be conducted to validate the theory in real outdoor environments with specific antennas.

\section{ACKNOWLEDGMENT}

The authors thank the UIC Machine shop for manufacturing the scaled models. The authors are thankful to the Reviewers 


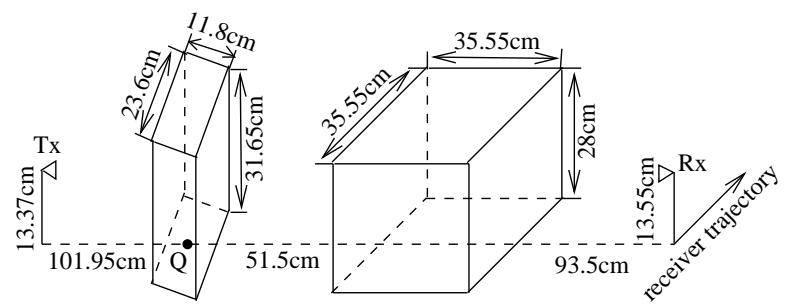

(a) Two-buildings block, the small box rotated $20^{\circ}$ clockwise

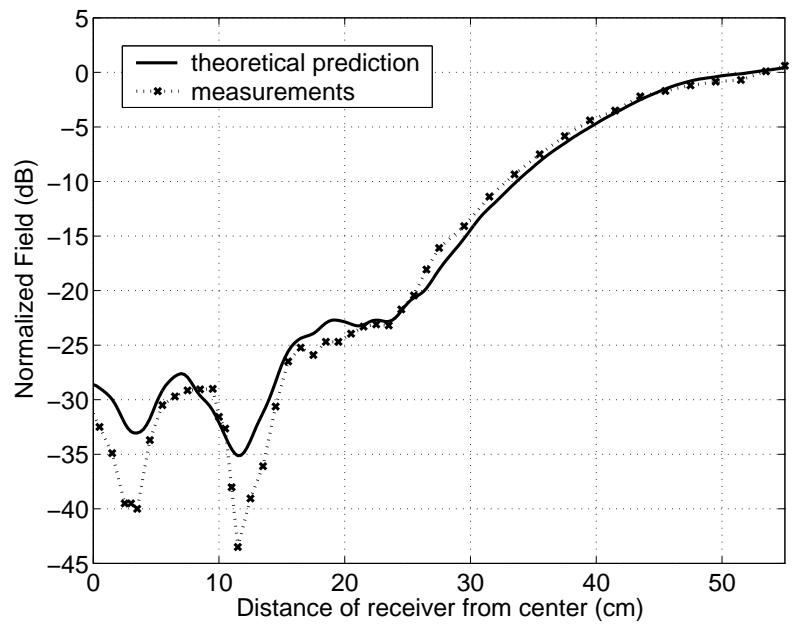

(b) Normalized field, vertical polarization

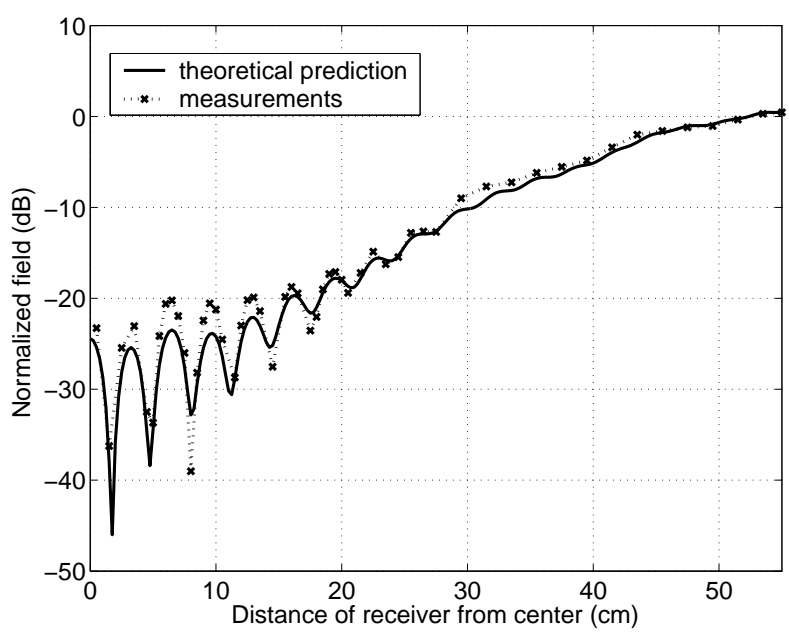

(c) Normalized field, horizontal polarization

Fig. 11. Geometry and normalized field for two buildings, with the small box rotated $20^{0}$ clockwise

for suggesting improvements to this manuscript.

\section{REFERENCES}

[1] Y. Okumura, E. Ohmori, T. Kawano, and K. Fukuda, "Field strength and its variability in VHF and UHF land-mobile radio service," Rev. Elec. Commun. Lab., vol. 16, pp. 825-873, 1968.

[2] M. Hata, "Empirical formula for propagation loss in land mobile radio services," IEEE Trans. Veh. Technol., vol. VT-29, pp. 317-325, Aug. 1980.

[3] G. L. Turin, F. F. Clapp, T. L. Johnston, S. B. Fine, and D. Lavry, "A statistical model of urban multipath propagation," IEEE Trans. Veh. Technol., vol. VT-21, pp. 1-9, Feb. 1972.

[4] H. Suzuki, "A statistical model for urban radio propagation," IEEE Trans. Commun., vol. COM-25, pp. 673-680, July 1977.

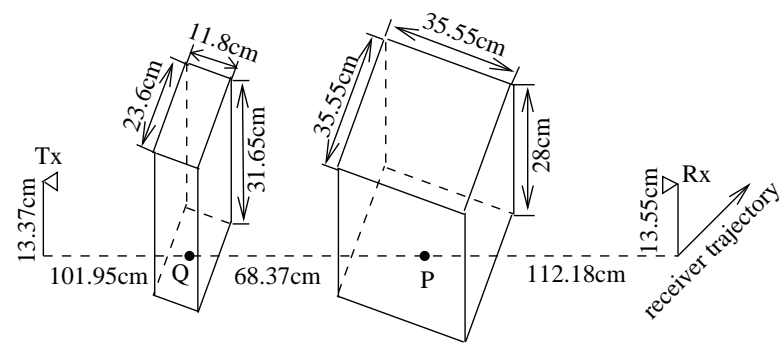

(a) Two-buildings block, both rotated $20^{\circ}$ clockwise

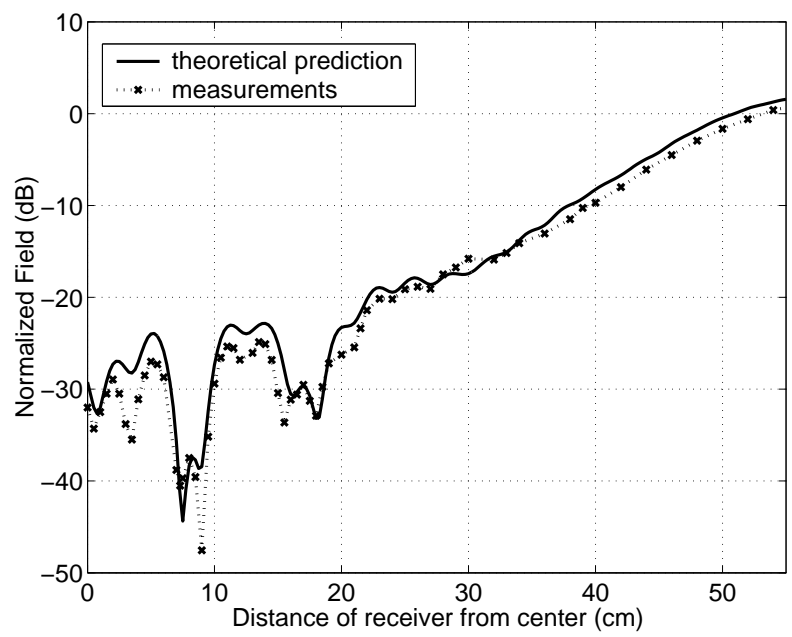

(b) Normalized field, vertical polarization

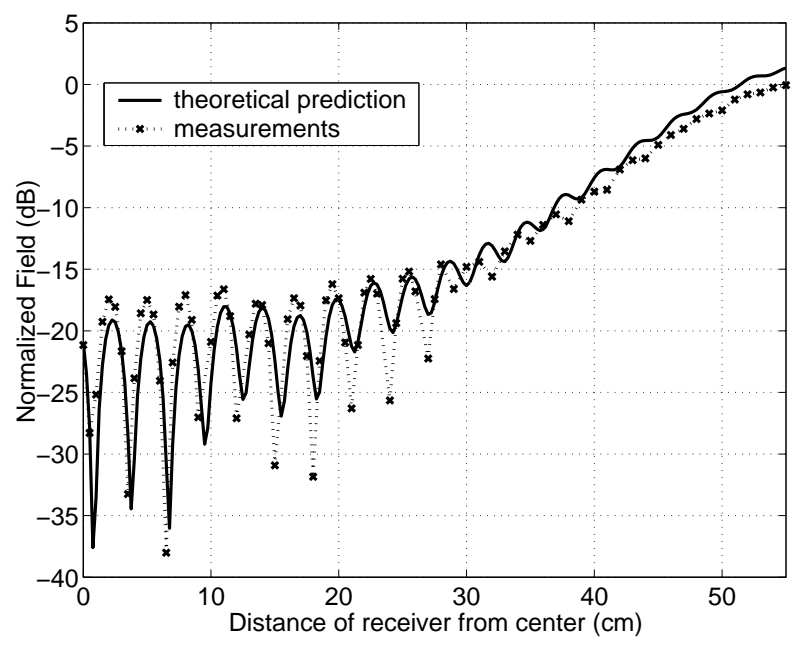

(c) Normalized field, horizontal polarization

Fig. 12. Geometry and normalized field for two buildings, both boxes rotated $20^{0}$ clockwise

[5] F. A. Agelet, F. P. Fontan, and A. Formella, "Fast ray tracing for microcellular and indoor environments," IEEE Trans. Magn., vol. 33, no. 2, pp. 1484-1487, 1997.

[6] G. Liang and H. L. Bertoni, "A new approach to 3-D ray tracing for propagation prediction in cities," IEEE Trans. Antennas Propagat., vol. 46, no. 6, pp. 853-863, June 1998.

[7] Z. Q. Yun, Z. J. Zhang, and M. F. Iskander, "A ray-tracing method based on the triangular grid approach and application to propagation prediction in urban environments," IEEE Trans. Antennas Propagat., vol. 50, no. 5, pp. 750-758, May 2002.

[8] D. Erricolo and P. L. E. Uslenghi, "Two-dimensional simulator for propagation in urban environments," IEEE Trans. Veh. Technol., vol. 50, pp. 1158-1168, July 2001. 
[9] L. Saini and U. Casiraghi, "A 3D fourier split-step technique for modeling microwave propagation in urban areas," in Fourth European Conference on Radio Relay Systems, pp. 210-214, 1993.

[10] J. H. Whitteker, "Fresnel-kirchhoff theory applied to terrain diffraction problems," Radio Sci., vol. 25, no. 5, pp. 837-851, 1990.

[11] L. C. Ong and C. C. Constantinou, "Urban radiowave propagation: a 3-D path-integral wave analysis," IEEE Trans. Antennas Propagat., vol. 46, pp. 211-217, 1998.

[12] R. S. Kirby, H. T. Dougherty, and P. L. McQuate, "Obstacle gain measurements over pikes peak at 60 to 1,046 Mc," Proc. IRE, vol. 43 , pp. $1467-1472,1955$

[13] G. Millington, R. Hewitt, and F. S. Immirzi, "Double knife-edge diffraction in field-strength predictions," Proc. Inst. Electr. Eng., Monograph 507E, pp. 419-429, 1962.

[14] K. Furutsu, "On the theory of radio wave propagation over inhomogeneous earth," J. Res. Nat. Bur. Stand., 67D, pp.39-62, 1963.

[15] L. E. Vogler, "An attenuation function for multiple knife-edge diffraction," Radio Sci., vol. 17, pp. 1541-1546, 1982.

[16] J. J. Stamnes, B. Spjelkavik, and H. M. Pedersen, "Evaluation of diffraction integrals using local phase and amplitude approximations," Opt. Acta., vol. 30, no. 2, pp. 207-222, 1983.

[17] J. Walfisch and H. L. Bertoni, "A theoretical model of UHF propagation in urban environments," IEEE Trans. Antennas Propagat., vol. 36, no. 12, pp. 1788-1796, Dec. 1988.

[18] M. S. Assis, "Effect of lateral profile on diffraction by natural obstacles," Radio Sci., vol. 17, no. 5, pp. 1051-1054, Sept.-Oct. 1982.

[19] S. V. Savov, J. H. Whitteker, and R. Vasilev, "Attenuation of waves behind a building," IEE Proc. Microw. Antennas Propag., vol. 146, no. 2, pp. 145-149, Apr. 1999.

[20] C. J. Haslett, "Modelling and measurements of the diffraction of microwaves by buildings," IEE Proc. Microw. Antennas Progag., vol. 141, no. 5, pp. 397-401, Oct. 1994.

[21] T. A. Russell, C. W. Bostian, and T. S. Rappaport, "A deterministic approach to predicting microwave diffraction by buildings for microcellular systems," IEEE Trans. Antennas Propagat., vol. 41, no. 12, pp. 16401649, Dec. 1993.

[22] M. F. Levy, Parabolic equation methods for electromagnetic wave propagation, London: IEE Press, 2000.

[23] R. Janaswamy, "Path loss predictions in the presence of buildings on flat terrain: a 3D vector parabolic equation approach," IEEE Trans. Antennas Propagat., vol. 51, no. 8, pp. 1716-1728, Aug. 2003.

[24] D. E. Eliades, "Path integral analysis of paraxial radiowaves propagation over a nonlevel plateau," Proc. Inst. Elect. Eng., vol. 138, part. H, pp. 521-526, Dec. 1991.

[25] C. Brennan, and P. J. Cullen, "Application of the fast far-field approximation to the computation of UHF pathloss over irregular terrain," IEEE Trans. Antennas Propagat., vol. 46, no. 6, pp. 881-890, June 1998

[26] C. Brennan, P. J. Cullen, and L. Rossi, "An MFIE-based tabulated interaction method for UHF terrain propagation problems," IEEE Trans. Antennas Propagat., vol. 48, no. 6, pp. 1003-1005, June 2000.

[27] J. T. Hviid, J. Bach Andersen, J. Toftgård, and J. Bøjer, "Terrain-based propagation model for rural area - An integral equation approach," IEEE Trans. Antennas Propagat., vol. 43, no. 1, pp. 41-46, Jan. 1995.

[28] R. P. Feynman and A. R. Hibbs, Quantum mechanics and path integrals, McGraw-Hill, New York, 1965.

[29] S. W. Lee, "Path integrals for solving some eletromagnetic edge diffraction problems," J. Math. Phys., vol. 19, pp. 1414-1422, 1978.

[30] C. C. Constantinou and L. C. Ong, "Use of zero vs. nonzero thickness diffracting obstacles in radio channel modelling," 5th IEEE Int. Symp. PIMRC, pp. 280-286, Sept. 1994.

[31] D. Erricolo, G. D'Elia, and P. L .E. Uslenghi, "Measurements on scaled models of urban environments and comparisons with ray-tracing propagation simulation," IEEE Trans. Antennas Propagat., vol. 50, no. 5, pp. 727-735, May 2002.

[32] D. Erricolo, U. G. Crovella, and P. L. E. Uslenghi, "Time-domain analysis of measurements on scaled urban models with comparisons to ray-tracing propagation simulation," IEEE Trans. Antennas Propagat., vol. 50, no. 5, pp. 736-741, May 2002.

[33] Y. Xu, Q. Tan, D. Erricolo, and P. L. E. Uslenghi, "3D propagation in urban environments using Fresnel-Kirchhoff integrals," IEEE Antennas and Propagation Society Topical Conference on Wireless Communication Technology, Honolulu, Hawaii, Oct. 2003.

[34] D. Erricolo, "Measurements in anechoic chambers to validate wireless propagation models," International Symposium on Electromagnetic Compatibility EMC Europe 2002, Vol. 2, pp. 653-658, Sorrento, Italy, Sept. 9-13, 2002
[35] G. A. J. van Dooren and M. H. A. J. Herben, "Polarisation-dependent site-shielding factor of block-shaped obstacle," Electron. Lett., vol. 29, pp. 15-16, 1993.

[36] Y. Xu, "Fresnel-Kirchhoff integral for path loss prediction in outdoor urban environments," Ph.D. dissertation, Univ. of Illinois at Chicago, 2004.

[37] M. Born and E. Wolf, Principles of Optics, 3rd ed. New York: Pergamon, 1965.

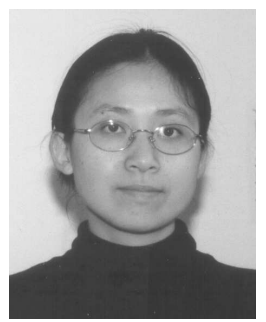

Ying $\mathrm{Xu}$ received the B.S. in electrical engineering from Wuhan University, China, in 1994, and the M.S. in electrical engineering from Beijing Institute of Environmental Features, China, in 1997, and the $\mathrm{Ph} . \mathrm{D}$. degree at the University of Illinois at Chicago, in 2004 .

Her research interests include computational electromagnetics and radio wave propagation in wireless communications.

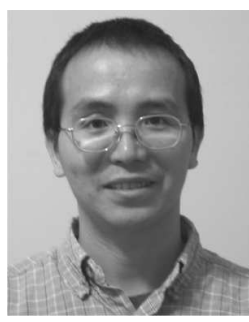

Qiwu Tan (S'02-M'05) was born in Hunan province, China, in 1969. He received the Bachelor and Master degrees from Xidian University, Xi'an, China, in 1991 and 1994, respectively, and the Ph.D. degree from the University of Illinois at Chicago in 2004, all in electrical engineering.

From 1994 to 1999, he was an electrical engineer working on phased array in Beijing Institute of Radio Measurement, China Aerospace Industry Corporation (China Aerospace Administration). During that period, he was honored one first and two second prizes of Science \& Technology Progress awarded by China Aerospace Industry Corporation and one third prize of National Science \& Technology Progress awarded by the Department of Science \& Technology, China. In 2004, he joined Amphenol T\&M Antennas designing antennas, including external and internal antennas, for mobile phone, WLAN, GPS and bluetooth applications.

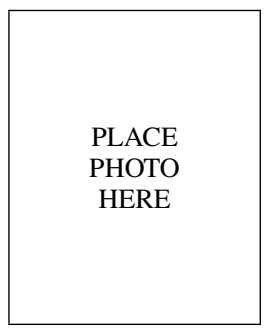

Danilo Erricolo

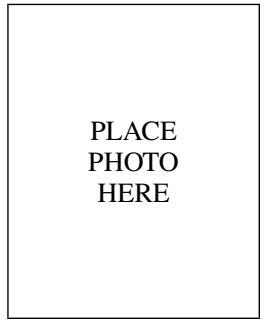

Piergiorgio L.E. Uslenghi 